\title{
Entrenamiento de recursos humanos para la gerencia de calidad en el sector de la salud
}

\section{Training of human resources for quality management in the health sector}

DOI: http://dx.doi.org/10.17981/cultedusoc.11.2.2020.18

Recibido: 27 de abril de 2020 Aceptado: 26 de junio de 2020 Publicado: 12 de julio de 2020

\author{
Remedios Pitre Redondo \\ Universidad de la Guajira. Riohacha (Colombia) \\ rpitre@uniguajira.edu.co \\ Johennys Genith Brito Chiquillo \\ Universidad de la Guajira. Riohacha (Colombia) \\ johennys.brito@gmail.com \\ Hugo Hernández Palma \\ Universidad del Atlántico. Barranquilla (Colombia) \\ hugohernandezp@mail.uniatlantico.edu.co
}

Para citar este artículo:

Pitre, R. Brito, J. y Hernández, H. (2020). Entrenamiento de recursos humanos para la gerencia de calidad en el sector de la salud. Cultura, Educación y Sociedad, 11(2). 285-302. DOI: http://dx.doi.org/10.17981/cultedusoc.11.2.2020.18

\section{Resumen}

Para el sector de la salud, uno de los factores más importantes es el recurso humano, por lo que la inversión realizada en este ámbito es muy positiva en términos de apropiación de objetivos, cultura laboral, innovación o productividad. Este artículo se orienta a analizar el proceso de capacitación y evaluación del personal de salud en Colombia. Para la investigación propuesta, se eligió una metodología cuantitativa que, apoyada en la revisión de la literatura, facilitó la abstracción de las categorías centrales relacionadas con el tema y permitió, al final, hacer reflexiones centradas en la mejora. Los resultados permiten reconocer la importancia de gestionar y desarrollar los recursos humanos involucrados en los procesos organizacionales para optimizar el logro de las metas. Se concluye que el recurso humano es la clave para el logro de la calidad dentro de las organizaciones. Palabras clave: Salud; recursos humanos; mejora continua; calidad

\begin{abstract}
For the health sector, one of the most important factors is human resources, so the investment made in this area is very positive in terms of appropriation of objectives, work culture, innovation or productivity. This article aims to analyze the training and evaluation process of health personnel in Colombia. For the proposed research, a quantitative methodology was chosen that, supported by the literature review, facilitated the abstraction of the central categories related to the subject and the policies, in the end, making reflections focused on improvement. The results recognized the importance of managing and developing the human resources involved in the organizational processes to achieve the goals. It is concluded that human resources are the key to achieving quality within organizations.
\end{abstract}

Keywords: Health; human resources; continuous improvement; quality 


\section{INTRODUCCIÓN}

Las instituciones sanitarias (IPS) son entidades que forman parte del sistema general de salud, ofreciendo un servicio crítico para la percepción del bienestar de sus usuarios; en este sentido, la implantación de sistemas de gestión es conveniente para este tipo de organización ya que tiene la responsabilidad de garantizar la seguridad del paciente (Graban, 2016), al mismo tiempo que requiere aumentar la productividad y aprovechar recursos disponibles (Arenas y Tamayo, 2010). Comprender la calidad como la idoneidad de un servicio para satisfacer o superar las expectativas de un usuario o cliente (Mitra, 2016), el esfuerzo por mejorar continuamente sus procesos debe ir acompañado de estrategias encaminadas a aumentar el rendimiento de los recursos humanos, que es fundamental para mejorar la calidad de la atención médica y la seguridad del paciente (Carayon et al., 2014).

Los beneficios proporcionados por los programas de capacitación y desarrollo como parte de los sistemas de calidad en las organizaciones son de gran beneficio para mejorar la gestión y obtener resultados favorables en términos de satisfacción del cliente y generación de dividendos (Phillips, 2012); a medida que los empleados son instruidos y alineados con las estrategias corporativas, su trabajo genera una mayor contribución al logro de los objetivos globales (Ramazani \& Jergeas, 2015); siendo parte de un complejo proceso de planificación estratégica (Niebles, Torres, Núñez \& Villa, 2019).

Para las instituciones de salud, este aspecto es de gran valor, teniendo en cuenta que el desarrollo de su negocio se basa en el trabajo de un gran número de profesionales de diversa naturaleza, que están en contacto permanente con sus usuarios, por lo que es posible para ellos establecer el tipo de expectativas que traen, y cómo pueden estructurar su trabajo para lograr la satisfacción del servicio deseado (Al-Abri y Al-Balushi, 2014).

Con base a lo explicado anteriormente, se menciona que el presente artículo de investigación se direcciona a analizar el proceso de capacitación y evaluación del personal de salud en Colombia. Para la consecución del objetivo planteado se proponen las siguientes categorías de apartados del articulo: Metodología, Resultados con las categorías de Antecedentes de la gerencia en el sector salud y el Contexto de entrenamiento de RRHH en el sector salud; seguidos de las conclusiones de estudio.

\section{Metodología}

La metodología desarrollada en el presente artículo de investigación se basa en el enfoque cualitativo; siendo este un estudio de tipo documental en el cual se dio uso de una revisión de literatura de manera que se lograse obtener la información necesaria para llevar a cabo un análisis de contenido de las fuentes de información seleccionadas. Sobre las fuentes de información, se destaca que estas fueron seleccionadas de plataformas como Google Academico, Scielo, Redalyc y Scopus.

El análisis de contenido es un método de análisis de información cualitativo, el cual se direcciona en establecer una serie de categorías de análisis sobre la data recolectada. De este modo las categorías desarrolladas son: a) Antecedentes de la gerencia en el sector salud y b) Contexto de entrenamiento de RRHH en el sector salud; seguidos de las conclusiones de estudio. 


\section{Resultados}

Antecedentes de la gerencia en el sector salud

Desde 1993, cuando se promulgó la Ley 100 (1993), se establecieron las funciones y responsabilidades atribuibles a las instituciones de atención de la salud (IPS). Sin embargo, todavía existen problemas relacionados con la integralidad, eficacia y eficiencia del servicio prestado, que han llevado a muchas instituciones a una profunda crisis que afecta no sólo a los propietarios de estas entidades, sino también a los usuarios del sistema general de salud (Peña y Pineda, 2013).

La implementación de sistemas con modelos basados en la gestión de procesos representa una oportunidad para generar valor en las empresas (Vom Brocke, Petry \& Gonser, 2016). Su base principal es la implementación de una estrategia integral con énfasis en la mejora continua (Hammer, 2015), con el fin de proporcionar a los clientes una mayor fiabilidad en cuanto al cumplimiento de los requisitos, mayores niveles de rendimiento, reducción de costos, reducción de inventarios, logística más eficiente y mayor rendimiento operativo (Evans \& Lindsay, 2013).

TABLA 1.

Aspectos comunes en la medición de desempeño general de entidades de salud

\begin{tabular}{|c|c|}
\hline Categorías & Aspecto \\
\hline \multirow{3}{*}{ Acceso y Sensibilidad } & Disponibilidad \\
\hline & Oportunidad de Servicio \\
\hline & Hospitalidad \\
\hline \multirow{4}{*}{ Calidad } & Servicios Integrales \\
\hline & Exactitud del Diagnostico \\
\hline & Estándares de Gerencia \\
\hline & Retención del Cliente \\
\hline \multirow{4}{*}{ Resultados } & Tasas del éxito del tratamiento \\
\hline & Cobertura poblacional \\
\hline & Morbosidad \\
\hline & Mortalidad \\
\hline \multirow{3}{*}{ Responsabilidad, Transparencia y Regulación } & Accesibilidad y calidad de Información \\
\hline & Funciones de Salud Pública \\
\hline & Capacidad Reformada \\
\hline \multirow{2}{*}{ Justicia y Equidad } & Barreras Financieras para el Cuidado \\
\hline & Justicia Distributiva \\
\hline \multirow{3}{*}{ Eficiencia } & Costos \\
\hline & Excesos \\
\hline & Retrasos \\
\hline
\end{tabular}

Fuente: Adaptada de Basu \& Wright, 2012. 
Los beneficios que aporta la implantación de este tipo de gestión lo hacen óptimo para las empresas dedicadas a la prestación de servicios sanitarios (Ginter, Duncan \& Swayne, 2018), porque la naturaleza de este negocio y el sistema en el que está inmerso, están obligados a mejorar permanentemente la eficacia de sus procesos (Iden, 2012). En estos casos, a pesar de que el cliente no elige el servicio que recibe, determina su nivel de satisfacción, especialmente en términos de tiempo y la eficacia del tratamiento utilizado para lograr la recuperación en salud (Serrano y Ortíz, 2012). En consecuencia, algunos de los aspectos más utilizados para medir el rendimiento operativo de las instituciones de atención de la salud se presentan en la Tabla 1.

Los procesos gestionados bajo el enfoque de calidad buscan desarrollarse con el objetivo de aumentar la competitividad organizacional (Elshaer \& Augustyn, 2016; Kafetzopoulos, Gotzamani \& Gkana, 2015) a través de la mejora continua de la calidad en todos sus componentes, que se muestra en la Figura 1 y que pueden resumirse en productos, servicios, personas, procesos y entornos de trabajo (Meyers, Durlak \& Wandersman, 2012). Esto demuestra que la calidad no se puede obtener tratando un aspecto individualmente; por el contrario, se trata de comprender la interrelación y la integralidad que existen entre estos elementos para mejorar su rendimiento como sistema (Goetsch \& Davis, 2014). A continuación, se observan las características de procesos gerenciales basados en calidad:

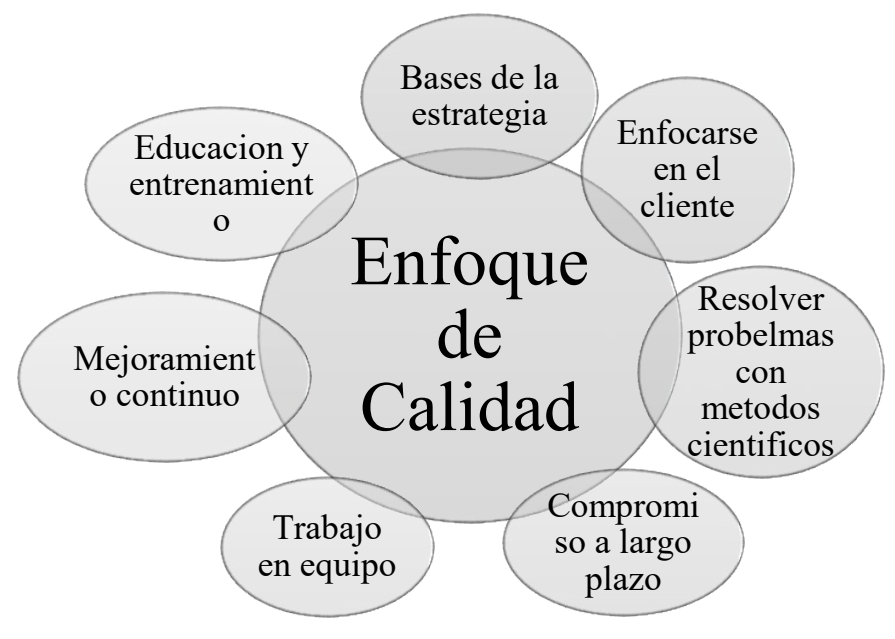

Figura 1. Características de procesos gerenciales basados en calidad Fuente: Autores.

Por su parte, Edward Deming (Coonor, 1986), uno de los mayores exponentes en cuanto a la aplicabilidad de la calidad en las organizaciones, determinó 14 puntos universales que deben abordarse en la administración para el máximo uso de conceptos relacionados con la calidad en los procesos, bienes y servicios, los cuales son (Ross, 2017):

- Crear conciencia de propósito con un plan: esto permite que los miembros del equipo de trabajo entiendan el porqué de dicho y plan y como este contribuyen al crecimiento de la organización, a su vez de como su rol influye en dicho proceso (Hernández, 2011).

- Adoptando la nueva filosofía de calidad: La filosofía de calidad permite que desde la alta gerencia hasta el cargo más bajo de la misma se tenga una verdadera conciencia (Palma, 2011). 
- Deje de confiar en la inspección masiva: pues esta se realiza de manera generalizada y no permite atender a las cuestiones más puntuales que muchas veces son las más descuidadas (Hernández, Martínez y Cardona, 2016).

- Finalizar la práctica de elegir proveedores basados en el precio: Pues se debe tener una relación equilibrada entre la calidad y el precio, de modo que no se comprometa la misma calidad que la organización ofrece a sus usuarios (Hernández et al., 2016).

- Identificar los problemas y el trabajo permanente necesario para la mejora del sistema: Todo sistema debe ser atendido de manera constante para no desmejorar la calidad de este (Hernández et al., 2016).

- Adoptar métodos modernos de formación en el trabajo: Cada vez la sociedad se mueve a una velocidad cada vez mayores y así son los procesos formativos, de modo que el entrenamiento del personal debe responder a la realidad actual (Hernández et al., 2016).

- Cambiar el enfoque de la producción orientada a más calidad en lugar de más cantidad: Muchas organizaciones consideran que más es menos, pero los mismos preceptos de la administración básica permiten reconocer que la calidad debe ir siempre por enciman de la cantidad (Hernández y Jiménez, 2016).

- Lidiar con el miedo: Este es un elemento constante en las todas las acciones de las organizaciones, especialmente en el sector salud por lo que es fundamental que los colaboradores y la alta gerencia sepan como trabajar con esta. (Hernández, 2017).

- Derribar barreras entre departamentos: Los departamentos no pueden ser vistos como sistemas cerrados, pues esto no permitirá una verdadera cohesión que facilite logra la calidad organizacional (Hernández, 2017).

- Detener la demanda de aumentar la calidad sin proporcionar los métodos necesarios para lograrlos: la calidad debe venir de un proceso lógico de planeación, ejecución y evaluación (Hernández, 2017).

- Eliminar los estándares de trabajo basados en cuotas numéricas: Se debe reconocer que el trabajo muchas veces no puede ser medido en escalas cuantificables, donde los datos cualitativos o cuanti-cualitativos brindan una mejor visión de la realidad (Jiménez, Hernández, Hernández, Cabas \& Ferreira, 2018).

- Eliminar las barreras generadas por el orgullo en la fuerza de trabajo: Las barreras impuestas en los sitios de trabajo son reconocidas como un gran obstáculo para la calidad en las organizaciones (Hernández, Martínez y Rodríguez, 2017).

- Crear una estructura en la alta administración que tienda para el cumplimiento de los 13 puntos anteriores: como se ha mencionado, la calidad debe ser asumida desde los altos cargos de la organización pues son estos quienes le dan longevidad a dichos sistemas (Hernández y Jiménez, 2016).

Como se puede observar en los fundamentos de la calidad, un gran número de aspectos implican el componente del factor humano en el desarrollo del servicio, que es congruente con la naturaleza del negocio (Albrecht, Bakker, Gruman, Macey \& Saks, 2015); así es como 
la inversión en este recurso permite obtener un rendimiento muy favorable en términos de la calidad del producto generado (Dale \& Plunkett, 2017). En esta línea de análisis, Dixon-Woods, McNichol y Martin (2012), llevaron a cabo investigaciones para identificar 10 aspectos esenciales que deben abordarse con el fin de mejorar la calidad de los servicios de salud a través de la intervención de recursos humanos (Figura 2).

\begin{tabular}{|c|c|}
\hline 1 & - Sensibilizar a la gente sobre la relevancia de los problemas existentes.. \\
\hline 2 & - Aceptacion de las soluciones propuestas como la mejor alternativa. \\
\hline 3 & $\begin{array}{l}\text { - Logro del funcionamiento óptimo de los sistemas de recolección y monitoreo de } \\
\text { datos. }\end{array}$ \\
\hline 4 & -Atención ante ambiciones y proyecciones excesivas. \\
\hline 5 & - Reforzar la cultura organizacional. \\
\hline 6 & • Evluar capacidades. \\
\hline 7 & - No tener compromiso del personal e incentivos para la partcipacion del personal. \\
\hline 8 & - Liderazgo. \\
\hline 9 & - El riesgo de consecuencias involuntarias. \\
\hline 10 & - Asegurar sustentabilidad. \\
\hline
\end{tabular}

Figura 2. Aspectos esenciales de la gerencia de recursos humanos para la gerencia de calidad Fuente: Adaptada de Dixon-Woods et al., 2012.

En este sentido, los sistemas de gestión de la calidad incluyen temas relacionados con el entrenamiento y la formación del personal con el fin de proporcionarles las herramientas de conocimiento, habilidades y aptitudes necesarias para lograr un mejor desempeño de sus funciones (Kumar, Maiti \& Gunasekaran, 2018); pieza clave para el éxito de las organizaciones (Niebles et al., 2019). Sin embargo, teniendo en cuenta el enfoque global que plantea el concepto de calidad en los procesos, es necesario que dicha planificación se lleve a cabo sobre la base de la calidad y los objetivos estratégicos estimulados hacia un horizonte de cumplimiento (Djordjevic, Panic, Arsic \& Zivkovic, 2018) de manera que sea posible crear mecanismos de control para determinar la eficacia y eficiencia de estas prácticas que están dando lugar a la mejora del rendimiento esperado (Wandersman, Chien \& Katz, 2012).

Es claro que la implementación de los sistemas de calidad genera un importante apoyo en los procesos de formación y entrenamiento de los trabajadores; los cuales son fundamentales para que las organizaciones sean capaces de cumplir sus metas de manera más efectiva, especialmente en el sector salud donde la calidad de la atención y de los procesos cobra un valor crítico. 


\section{Contexto de entrenamiento de RRHH en el sector salud}

La gestión del talento humano requiere una gestión adecuada, para ello, las evaluaciones y mediciones de su función de rendimiento como indicadores que demuestran la forma en que el personal lleva a cabo su trabajo y si está alineado con las expectativas las directivas, que también determinan la necesidad de formación y entrenamiento para la mejora de los servicios prestados. Implica a decir de Rodelo y Castro (2016), procesos de educacbilidad, formación y desarrollo humano.

Debido a lo anterior, entidades como el Instituto Mexicano del Seguro Social (IMSS), el mayor proveedor del sistema de salud en México, con más de 12 millones de beneficiarios, ha diseñado un plan de capacitación, en los últimos 25 años, en temas destacados en el área de emergencias y desastres, como apoyo vital avanzado en trauma; este programa de capacitación ha impactado a un total de 3015 médicos que recibieron el curso, los planes hospitalarios y la evacuación de unidades médicas, con 1792 personas capacitadas entre 2007 y 2014 (Cruz et al., 2016).

Por otro lado, se han incluido otros cursos internos para la evaluación de programas, como el Hospital Seguro, con un récord de 7474 capacitaciones; y el manejo de herramientas informáticas como el centro virtual de operaciones en emergencias y desastres, un curso que permite el desarrollo de habilidades como la comunicación desde zonas de emergencia, y que se dio a los profesionales de la salud de 1958; además, el curso con el mayor número de participantes fue soporte vital básico y avanzado con un porcentaje de 9728 personas capacitadas (Cruz, et al., 2016). De acuerdo con lo anterior, los principales impactos se muestran en la Figura 3.



Figura 3. Personal entrenado en apoyo básico y avanzado por ranking Fuente: Cruz et al., 2016.

En relación con la información proporcionada en la figura anterior, y de acuerdo con las cifras estimadas, el 70\% del personal capacitado son estudiantes, que incluyen, en su mayor parte, profesionales de la salud y personas especializadas en Apoyo Vital Avanzado; seguido por el equipo de profesionales, con un 18\%; proveedores de servicios, con una participación del 10\%; y por último, el equipo de instructores, con un porcentaje del $2 \%$ del total en formación recibida. 
Según los resultados de la investigación de Urdaneta y Urdaneta (2013), realizada en el Instituto de Investigaciones Biológicas, Clínicas y Medicina e Higiene en el Trabajo y la Investigación Dental de la Universidad de Zulia (Venezuela), la actuación formal y no formal sistema de evaluación aplicado al personal es poco frecuente; Además, de acuerdo con los resultados de las encuestas realizadas, el 55,80\% del personal afirma que recibe comentarios de su jefe sobre su desempeño dentro de la institución; 13.7\% declara que no se corrigen en el trabajo realizado. El 11,6\% confirmó que ha tenido evaluaciones escritas; y, en menor porcentaje, se confirma que se celebran reuniones periódicas, aunque con poca periodicidad.

Sin embargo, no existe un sistema formal de evaluación que identifique reestructuraciones positivas de los procesos y comentarios sobre las situaciones presentadas en busca de mejoras a corto y/o a largo plazo para fortalecer la calidad de los servicios prestados en el sector de la salud. El caso del Centro Ambulatorio de Cienfuegos coincide con la falta de reconocimiento de tareas que han sido bien ejecutadas (Urdaneta y Urdaneta, 2013).

El programa Hospital Seguro, en México, desarrolla un curso virtual sobre temas de revisión de los contenidos del programa, como el sistema nacional de protección civil, gestión de riesgos, índice de seguridad hospitalaria, evacuación hospitalaria, entre otros, que luego se evalúan en detalle con el personal elegido entre médicos, paramédicos, personal técnico y administrativo; como resultado de esta actividad, de 1323 personas inscritas, sólo 298 pasaron el curso con una nota media de 8,85; Como resultado, el curso, cuyo objetivo es la preparación para la respuesta de emergencia, ni siquiera obtuvo el 30\% del personal aprobado, lo que demuestra la necesidad de buscar las principales causas del bajo rendimiento de la mayoría del personal registrado, en la medida en que repercusiones en la falta de preparación del personal sanitario que debe comprender obligatoriamente estas cuestiones prioritarias en caso de respuesta inmediata a una contingencia (Cruz et al., 2018).

Por otro lado, El Programa de Emergencias y Desastres de la Representación en Colombia se destacó entre varios países al ganar el premio a buenas prácticas de maternidad seguras, una vez que el tema fue revisado en 93 casos de 19 países, lo que resultó en Colombia y Uruguay destacados como primer lugar, con la propuesta de cambio de salud y, en segundo lugar, respectivamente, por la reducción del riesgo y el daño en relación con el aborto (Organización Panamericana de la Salud, OPS, 2019).

La sostenibilidad de estos programas se basa en la capacitación de parteras y la movilización de recursos que permiten a las futuras madres un mayor acceso al sistema de salud; sin embargo, en Colombia también hay un refuerzo en los aspectos generales de la gestión de riesgos, debido a la ausencia de profesionales capacitados en estos temas. En este sentido, y según el informe García (2014), el 73\% de los programas que incluyen el área de conocimiento en gestión de riesgos están formados por Ingeniería, Arquitectura y Urbanismo; y en porcentajes inferiores al 15\%, existen profesiones relacionadas con la medicina veterinaria, la salud, la educación y las ciencias naturales (García, 2014).

El análisis de mejora propuesto por García (2014) para aumentar la capacidad de respuesta e innovación en el sector de la salud, en la ciudad de Barranquilla, permite verificar la relevancia de la interacción de ciertos elementos internos que deben ser promovidos, 
algunos en mayor grado que otros. Esto ciertamente permite saber cuáles son los factores que poseen una mayor incidencia sobre la innovación para que la manipulación de estos permita optimizar los procesos para encaminarse a innovar.

Sin embargo, Barranquilla es considerada una de las principales ciudades del país con el desarrollo de servicios de calidad en el sector de la salud, debido a que en los años 90 fue el punto de ubicación estratégico para diferentes IPS, EPS, con una importante intervención de entidades reguladoras como la Cámara de Comercio y el Ministerio de Salud; teniendo en cuenta lo anterior, entre los elementos que permiten la mejora de la competitividad hay 19 variables que influyen directamente en el sistema de crecimiento, y de las cuales 4 son en su mayoría relevantes para la consecución de la mejora de la innovación de cualquier organización (Pinilla, Martínez y Cabarcas, 2017); estos aspectos se definen en la Figura 4.

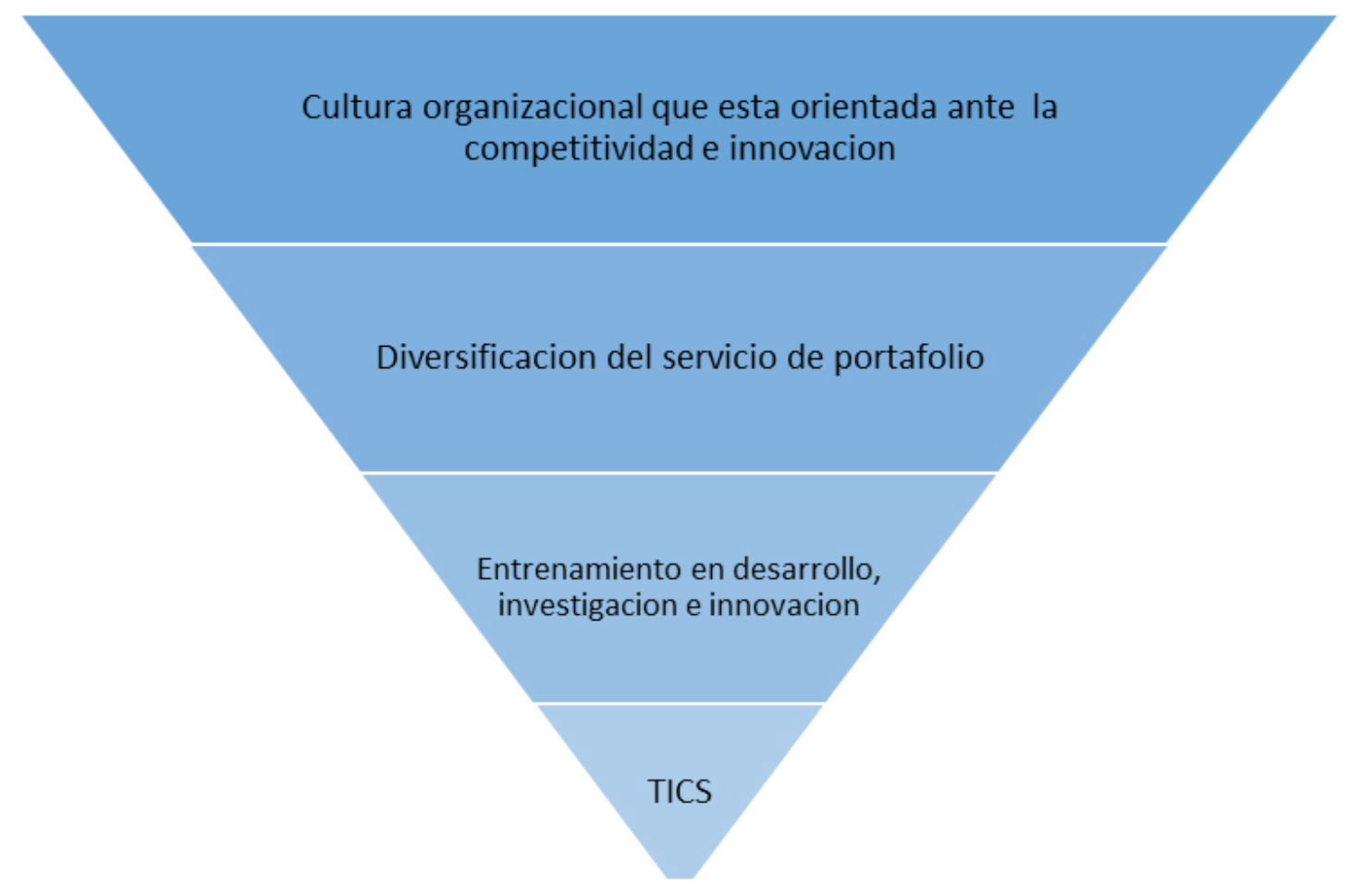

Figura 4. Variables Relevantes en el Sistema de Salud.

Fuente: Pinilla et al., 2017.

Según los elementos representados en la figura 4 de la investigación de Pinilla et al. (2017), donde fueron consultados por medio de entrevistas a 10 expertos del sistema de salud de la ciudad de Barranquilla, la cultura organizacional representa el lugar número uno dentro de la pirámide, representando un mayor grado de relevancia en la gestión de los procesos, influencia categorizada como gestión interna; sigue en segundo lugar la necesidad de diversificación de la cartera de servicios; en tercer lugar se encuentra la formación del personal, que debe establecerse como objetivo a nivel estratégico (Pinilla et al., 2017).

Por último, la gestión de las TIC, que también son gestionadas internamente por organizaciones de salud pública; Además, otras variables que actúan en menor proporción son la inversión privada en ciencia y tecnología, y las relaciones con entidades como el gobierno y las empresas, que ejercen una influencia externa para el empuje competitivo del sector 
de la salud. Cabe señalar que la categorización obtenida corresponde al resultado de la construcción de una matriz MIC MAC, propuesta por Pinilla et al. (2017), cuyo resultado cuantitativo generó mayor valor e importancia entre las variables encontradas en los primeros lugares de la pirámide presentada.

En relación con lo anterior, el Hospital Militar Central de Bogotá fue medido por sus trabajadores en el tema de capacitación, de acuerdo con el nivel de requerimiento planteado para la mejora de los servicios prestados, especialmente en el área de traumatología y altamente complejo patologías. Las prioridades de formación requeridas por el personal eran, en primer lugar, temas relacionados con el puesto ocupado, con un 32\%; aspectos jurídicos, con un 17\%; gestión de los conflictos internos y externos, con un 16\%; gestión de pacientes en crisis, con un 15\%; calidad, desde el 10\%; procesos administrativos, con un 5\%; archivos y gestión de documentos, con 4\%; y por último, el 3\% representa otros aspectos relacionados con la seguridad de los pacientes (HOMIL, 2015).

Por otro lado, con respecto a la capacitación en instituciones de salud de tercer nivel en Medellín, y con base al análisis del sujeto en entidades que pertenecen a las empresas estatales y a la Cooperativa de Hospitales Antioquia, se afirma que todavía existen deficiencias en áreas como el desarrollo, la inducción y la formación; en este sentido, la mayoría del personal, en más del 60\%, afirman que no tienen apoyo para seguir especializándose con estudios en el área que realizan; Además, en cursos cortos, en instituciones públicas y privadas, el 58\% del personal entrevistado confirmó que no ha recibido apoyo económico en este ámbito, algo que es relativamente preocupante debido a que este sector tiene intervención directa en la prevención y tratamiento de enfermedades para las comunidades (Arboleda, 2012).

A este respecto, hay que tener en cuenta que, en el caso del sector de la salud pública, el Gobierno de Colombia financia los recursos a través del presupuesto general de la nación (DNP, 2016). Sin embargo, según la investigación realizada sobre el presupuesto del Gobierno Nacional, y proporcionada por el Ministerio de Salud (Minsalud, 2016), las cifras de inversión en formación para 2016 correspondientes a la Tabla 2 son estructuradas de la siguiente manera.

TABLA 2.

Proyecciones de la Inversión en entrenamiento del Personal de la Salud

\begin{tabular}{lcc}
\hline & Destination Value & Reserved Value \\
\hline $\begin{array}{l}\text { Capacidades de reforzamiento para la planificación, vigilancia } \\
\text { y respuesta de la salud pública, y conocimiento gerencial a nivel } \\
\text { nacional. }\end{array}$ & $\$ 21.000 .000 .000$ & $\$ 1.392 .824 .525$ \\
$\begin{array}{l}\text { Implementación de estudios e investigación de las instituciones } \\
\text { de evaluación tecnológica de la salud a nivel nacional. }\end{array}$ & $\$ 3.500 .000 .000$ & $\$ 303.702 .326$ \\
$\begin{array}{l}\text { Implementación del currículo e investigación en el sector de } \\
\text { salud y protección social a nivel nacional. }\end{array}$ & $\$ 1.550 .000 .000$ & $\$ 132.572 .264$ \\
& $\$ 26.050 .000 .000$ & $\$ 1.829 .099 .115$ \\
\hline
\end{tabular}

Fuente: Ministerio de Salud, 2016. 
Como se puede ver en la Tabla 2 de las tres partidas asignadas por el gobierno a la inversión en proyectos de capacitación y generación de conocimiento en el sector de la salud, el presupuesto está liderado por el fortalecimiento de las capacidades de planificación, vigilancia y respuesta en salud, con $81 \%$; nuevos estudios e investigaciones a nivel nacional continúan con un 13\%; y en tercer lugar, el 6\% en otros estudios relativos al sector de la salud y la protección social; además, los valores reservados corresponden al 76\% en los casos en que se requiere cobertura adicional de la inversión, y al fortalecimiento de las capacidades de planificación en salud, vigilancia y respuesta; el 17\% restante y el $7 \%$ restante pueden utilizarse, en general, para estudios científicos. La Tabla 3 muestra los porcentajes de inversión en el presupuesto total destinado al sector de la salud por el Ministerio Nacional de Salud.

TABLA 3.

Presupuesto Nacional en \% asignado en 2016 para el entrenamiento e investigación en salud

\begin{tabular}{lll}
\hline & \% Destinado & \% Reservado \\
Presupuesto nacional para el entrenamiento e investigación & $7 \%$ & $8 \%$ \\
\hline
\end{tabular}

Fuente: Ministerio de Salud, 2016.

Como se puede ver en la figura anterior, al calcular el porcentaje de representación correspondiente a la capacitación en el sector de la salud en Colombia, las cifras se reducen; por esta razón, es necesario profundizar en la forma en que otros proyectos contribuyen en esta área para que la formación del personal logre un aumento representativo en esta cifra.

En Medellín, Colombia, se está avanzando en el diseño de herramientas informáticas aplicables a la unidad de cuidados intensivos del sector de la salud, dando como resultado software que tiene capacidad para simular 674 equipos, y con el que es posible medir el retraso en la formación, que a su vez varía según el tipo de personal que lo maneje; de tal manera que los que más se retrasan en el proceso son médicos, porque son la base de la unidad con más de 100 horas requeridas; después de las enfermeras y asistentes (Franco, Jaramillo y Barreneche, 2015).

Por otro lado, en Colombia, el panorama de la atención primaria de salud es liderado por Bucaramanga; por lo tanto, para la población de Bumanguesa es necesario vigilar constantemente el desempeño del personal de salud. Según esto, en una encuesta, 359 trabajadores y 102 supervisores dieron sus puntos de vista sobre diversos aspectos relacionados con el desempeño de sus funciones (Rodríguez-Villamizar, Vera-Cala, Rivera, Rojas y Uribe, 2017).

Este ejercicio incluyó a 59 empleados de IPS en Santander, y evaluó la competencia de Saber Hacer, en el que el 64\% declaró que tiene facilidades para el trabajo en equipo, el liderazgo y aplica valores como el respeto a la opinión; algo que sus supervisores reafirmaron y que validan, en el 94\%, la capacidad del personal para la discreción y confidencialidad en el trabajo (Rodríguez, Vera, Rivera, Rojas, Uribe, 2017).

Cabe señalar que los conocimientos técnicos son verificados por los supervisores, que confirman que, en un rango del $62 \%$ y el $78 \%$, la capacidad y competencia técnica de los 
profesionales de la salud es actual, sin embargo, hay deficiencias en la investigación en el área de la salud pública, con un valor preocupante del 53\% de los supervisores encuestados, lo que muestra la necesidad de capacitación para permitir que el personal relacionado fortalezca esta competencia.

Por último, en saber y hacer, los mismos trabajadores se evaluaron a sí mismos y todos ellos confirman, casi el 100\%, que cuentan con los conocimientos básicos necesarios para la prevención, la promoción de la salud y la atención primaria; Asimismo, los profesionales con los mejores conocimientos en el área de promoción y prevención son los dedicados a la profesión médica, seguidos por los dedicados a la nutrición (Rodríguez, Vera, Rivera, Rojas, Uribe, 2017). Otras ciudades, como Cali, son evaluadas por la misma población, cuyo porcentaje medido de satisfacción corresponde al $90 \%$ con respecto al cuidado; sin embargo, entre el 18 y el 36\% de los encuestados hicieron sugerencias con respecto a los inconvenientes presentados en relación con el tiempo incurrido en la espera del servicio, además de la calidad del sistema (Moral, et al., 2011).

La gestión de la calidad se trata de la integración de todas las funciones y procesos de la organización con el fin de lograr una mejora continua en la calidad de los bienes y servicios (Topalović, 2015; (Marín, Riquett, Pinto, Romero y Paredes, 2017), siempre teniendo en cuenta el objetivo principal de la satisfacción del cliente (Basu \& Wright, 2012; Klochkov, Klochkova, Volgina \& Dementiev, 2016). En un estudio desarrollado por Kim, Kumar y Kumar (2012) se encontró que la aplicación de las prácticas de QM a través de la gestión de procesos manifiesta una relación directa y positiva con la innovación incremental, radical y administrativa, mientras que la capacidad desarrollada bajo este enfoque puede desempeñar un papel vital en la identificación de rutinas, el establecimiento de una base de aprendizaje y el apoyo a actividades innovadoras.

Ahora bien, con el fin de mejorar la eficacia en la implementación de sistemas basados en la calidad, se considera importante gestionar y desarrollar el recurso humano involucrado en el desarrollo de actividades (Paillé, Chen, Boiral \& Jin, 2014); una inversión en este ámbito proporciona mecanismos para el desarrollo de un ser humano más adaptado a las políticas corporativas, así como herramientas de motivación y desarrollo que permitan aumentar las tasas de retención de los empleados, y en consecuencia, una disminución en costos de reclutamiento y contratación, sumados al desgaste de inducir a nuevos empleados a sus puestos de trabajo (Storey , 2014). Todo lo anterior permite al miembro del equipo de trabajo centrar sus esfuerzos en proporcionar lo mejor de sí mismo en términos de lograr los objetivos globales, además de buscar constantemente formas de aumentar la calidad de sus resultados.

\section{Conclusiones}

Con base a los resultados encontrados y analizados en el presente artículo, se concluye en primera instancia que el entrenamiento de recursos humanos dentro de cualquier organización es fundamental para el proceso estratégico de cualquier empresa, pues por medio de esto se logra preparar y formar a los nuevos trabajadores para que puedan incorporarse de la manera más efectiva al entorno organizacional. A su vez que facilita que los trabajadores siempre estén al día con las tendencias actuales de su área, y así cumplir con 
los estándares del mismo mercado. Esto cobra vital importancia en el sector salud, donde se trabajan con las vidas de los seres humanos y la presión y la calidad exigida son muy elevadas. Partiendo de lo anterior, la calidad es reconocida como el estándar de trabajo de las organizaciones del sector salud, las cuales están constantemente trabajando para mejorar la prestación de sus servicios a los usuarios que acudan a dichas instituciones de este mencionado sector.

Con relación al objetivo de trabajo de analizar el proceso de capacitación y evaluación del personal de salud en Colombia, se concluye que los procesos de capacitación del personal son cada vez más efectivos y enfocados hacia la calidad de los procesos organizacionales; estableciendo la calidad como una filosofía dentro de las mismas organizaciones. Esto también se observa en los procesos de evaluación, pues han evolucionado basándose en este nuevo estándar de calidad.

Los resultados permiten reconocer como en los procesos de evaluación de las organizaciones del sector salud se empieza a dar un importante entendimiento de cómo no todos los procesos son cuantificables y, por ende, requieren de una revisión basándose en la realidad de dichos procesos. A su vez, las nuevas tecnologías y la planificación son algunas de las más importantes herramientas en la capacitación del personal dentro de este sector.

Como cierre, se considera que el camino hacia la calidad en el sector salud en Colombia aun es largo, pero apoyándose en los procesos de entrenamiento y evaluación del personal es posible vincularse desde la gestión estratégica del talento humano a los objetivos organizacionales de manera una efectiva con la calidad como la base de trabajo.

\section{REFERENCIAS}

Al-Abri, R. \& Al-Balushi, A. (2014). Patient satisfaction survey as a tool towards quality improvement.Omanmedicaljournal,29(1),3-7.https://dx.doi.org/10.5001\%2Fomj.2014.02

Albrecht, S. L., Bakker, A. B., Gruman, J. A., Macey, W. H. \& Saks, A. M. (2015). Employee engagement, human resource management practices and competitive advantage: An integrated approach. Journal of Organizational Effectiveness: People and Performance, 2(1), 7-35. https://doi.org/10.1108/JOEPP-08-2014-0042

Arboleda, G. (2012). Ingreso y capacitación del personal vinculado a instituciones del tercer nivel de atención en salud. Medellín, Colombia. Revista Gerencia y Políticas de Salud, 11(23), 134-151. Disponible en https://revistas.javeriana.edu.co/index.php/gerepolsal/article/view/4468

Arenas, C. H. y Tamayo, C. M. (2010). Indicadores administrativos y financieros antes y después de la acreditación en las instituciones prestadoras de servicios de salud en Colombia. CES Medicina, 24(2), 9-20. Disponible en https://revistas.ces.edu.co/index. $\mathrm{php} / \mathrm{medicina/article/view/1219}$

Basu, R. \& Wright, J. N. (2012). Quality beyond six sigma. London: Routledge.

Carayon, P., Wetterneck, T. B., Rivera-Rodriguez, A. J., Hundt, A. S., Hoonakker, P., Holden, R. \& Gurses, A. P. (2014). Human factors systems approach to healthcare quality and patient safety. Applied ergonomics, 45(1), 14-25. https://doi.org/10.1016/j.apergo.2013.04.023 
Coonor, P. (1986). Quality, Productivity and Competitive Position, In, W. E. Deming, Quality and Reliability Engineering International, 2(4). https://doi.org/10.1002/ qre.4680020421

Cruz, F., Loría, J., Hernández, I., Franco, R., Ochoa, C. y Sánchez, V. (2016). Experiencia en capacitación en emergencias de la División de Proyectos Especiales en Salud, Instituto Mexicano del Seguro Social. Cirugía y Cirujanos, 84(2), 127-134. https://doi. org/10.1016/j.circir.2015.12.001

Cruz, F., Sánchez, J., Cortes, H, Loria, J. y Montes, E. (2018). Tecnologías de la información en la formación de evaluadores del Programa Hospital Seguro en México. Cirugia y cirujanos, 86(3), 237-243. Disponible en https://www.medigraphic.com/cgi-bin/ new/resumen.cgi?IDARTICULO=82988

Dale, B. G. \& Plunkett, J. J. (2017). Quality Costing. London: Routledge. https://doi. org/10.4324/9781315245515

Dixon-Woods, M., McNicol, S. \& Martin, G. (2012). Ten challenges in improving quality in healthcare: lessons from the Health Foundation's programme evaluations and relevant literature. BMJ Qual Saf, bmjqs-2011, 21(10), 876-884. http://dx.doi.org/10.1136/ bmjqs-2011-000760

Djordjevic, P., Panic, M., Arsic, S. \& Zivkovic, Z. (2018). Impact of leadership on strategic planning of quality. Total Quality Management \& Business Excellence, 31(5-6), 1-15. https://doi.org/10.1080/14783363.2018.1490176

Elshaer, I. A. \& Augustyn, M. M. (2016). Direct effects of quality management on competitive advantage. International Journal of Quality \& Reliability Management, 33(9), 1286-1310. https://doi.org/10.1108/IJQRM-07-2014-0086

Evans, J. R. \& Lindsay, W. M. (2013). Managing for quality and performance excellence. Boston: Cengage Learning.

Franco, G., Jaramillo, D. y Barreneche, J. (2015). Modelo de capacitación de tecnología biomédica para clínicas y hospitales de tercer nivel, enfocado en personal asistencial. Revista Ingeniería Biomédica, 9(18), 139-144. https://doi.org/10.24050/19099762. n18.2015.781

García, A. (2014). Fortalecimiento de la capacitación e investigación em gestión del riesgo en Colombia: Producto 2. Documento de análisis, junto con una propuesta técnica de programas de capacitación/investigación que se requieren en materia de Gestión del Riesgo de Desastres. Bogotá, D.C.: Comisión Nacional de Investigación de Riesgo de Desastres en Colombia. Recuperado de http://portal.gestiondelriesgo.gov.co/Documents/Comision\%20Investigacion\%20Gestion\%20Riesgo/Lineamientos\%20de\%20 investigaci\%C2\%A2n\%202\%20(Unesco).pdf

Ginter, P. M., Duncan, W. J. \& Swayne, L. E. (2018). The strategic management of health care organizations. New York: John Wiley \& Sons.

Goetsch, D. L. \& Davis, S. B. (2014). Quality management for organizational excellence. New York: Pearson.

Graban, M. (2016). Lean hospitals: improving quality, patient safety, and employee engagement. Boca Raton: CRC press. 
Hammer, M. (2015). What is business process management? In: J. vom Brocke \& M. Rosemann (Eds.), Handbook on business process management 1 (pp. 3-16). Berlin: Springer.

Hernández, H. (2017). Sistemas de gestión integrados en el sector salud para la optimización de la calidad en el departamento del atlántico. Dictamen Libre, (20), 99-106. Disponible en http://www.unilibrebaq.edu.co/ojsinvestigacion/index.php/dictamenlibre/article/view/859

Hernández, H. G. (2011). La gestión empresarial, un enfoque del siglo XX, desde las teorías administrativas científica, funcional, burocrática y de relaciones humanas. Escenarios, 9(1), 38-51. Disponible en http://hdl.handle.net/11619/1628

Hernández, H. y Jiménez, A. (2016). Estrategia para impulsar el crecimiento sostenido de las instituciones prestadoras de servicios en el Distrito de Barranquilla, Colombia. Sotavento MBA, (28), 34-43. https://doi.org/10.18601/01233734.n28.04

Hernández, H., Martínez, D. M. y Cardona, D. C. (2016). Enfoque basado en procesos como estrategia de dirección para las empresas de transformación. Saber, Ciencia y Libertad, 11(1), 141-150. https://doi.org/10.18041/2382-3240/saber.2016v11n1.499

Hernández, H., Martínez, D. y Rodríguez, J. (2017). Gestión de la calidad aplicada en el mejoramiento del sector universitario. Revista Espacios, 38(20). [Online]. Disponible en https:/www.revistaespacios.com/a17v38n20/a17v38n20p29.pdf

HOMIL. (2015). Plan Institucional de Capacitación Hospital Militar Central. [Online]. Disponible en https://www.hospitalmilitar.gov.co/index.php?idcategoria=11078

Iden, J. (2012). Investigating process management in firms with quality systems: a multi-case study. Business Process Management Journal, 18(1), 104-121. https://doi. org/10.1108/14637151211215037

Jiménez, G., Hernández, L., Hernández, H., Cabas, L. \& Ferreira, J. (2018). Evaluation of Quality Management for Strategic Decision Making in Companies in the Plastic Sector of the Colombian Caribbean Region Using the TQM Diagnostic Report and Data Analysis. In: HCI 2018, International Conference on Human-Computer Interaction (pp. 273-281). Springer, Cham. https://doi.org/10.1007/978-3-319-92285-0_38

Kafetzopoulos, D., Gotzamani, K. \& Gkana, V. (2015). Relationship between quality management, innovation and competitiveness. Evidence from Greek companies. Journal of Manufacturing Technology Management, 26(8), 1177-1200. https://doi.org/10.1108/ JMTM-02-2015-0007

Kim, D.-Y., Kumar, V. \& Kumar, U. (2012). Relationship between quality management practices and innovation. Journal of operations management, 30(4), 295-315. https:// doi.org/10.1016/j.jom.2012.02.003

Klochkov, Y., Klochkova, E., Volgina, A. \& Dementiev, S. (2016). Human factor in quality function deployment. In: SMRLO, 2016 Second International Symposium on Stochastic Models in Reliability Engineering, Life Science and Operations Management (pp. 466-468). Beer-Sheva, Israel, https://doi.org/10.1109/SMRLO.2016.81

Kumar, P., Maiti, J. \& Gunasekaran, A. (2018). Impact of quality management systems on firm performance. International Journal of Quality \& Reliability Management, 35(5), 1034-1059. https://doi.org/10.1108/IJQRM-02-2017-0030 
Marín, F., Riquett, M., Pinto, M., Romero, S. y Paredes, A. (2017). Gestión Participativa y calidad educativa en el contexto del plan de mejoramiento institucional en Escuelas Colombianas. Opción, 33(82). 344-365. Disponible en https://produccioncientificaluz. org/index.php/opcion/article/view/22850

Meyers, D. C., Durlak, J. A. \& Wandersman, A. (2012). The quality implementation framework: a synthesis of critical steps in the implementation process. American journal of community psychology, 50(3-4), 462-480. https://doi.org/10.1007/s10464-012-9522-x

Mitra, A. (2016). Fundamentals of quality control and improvement. New York: John Wiley \& Sons.

Moral, R., Dios, A., Jiménez, C., González, V., García, M., Pérula, L. y Barrios, L. (2011). Percepción de los pacientes sobre la atención recibida por el personal no sanitario en los centros de salud. Revista de Calidad Asistencial, 26(2), 97-103. https://doi. org/10.1016/j.cali.2010.10.001

Niebles, L. D., Torres, K. M., Núñez, W. A. y Villa, M. L. (2019). Estrategias de marketing como herramienta para la gestión del desempeño. Barranquilla: Corporación Universitaria Latinoamericana. Disponible en https://libros.ul.edu.co/index.php/libros/cata$\log /$ book $/ 23$

OPS. (2019). El Programa de Emergencias y Desastres de la Representación en Colombia obtiene Premio en el V Concurso de Buenas Prácticas sobre maternidad segura con perspectiva de género. [Online]. Disponible en https://www.paho.org/col/ index.php?option=com_content\&view=article\&id=1452:el-programa-emergencias-ydesastres-representacion-en-colombia-obtiene-premio-en-v-concurso-buenas-practicas-sobre-maternidad-segura-con-perspectiva-genero\&Itemid=442

Paillé, P., Chen, Y., Boiral, O. \& Jin, J. (2014). The impact of human resource management on environmental performance: An employee-level study. Journal of Business Ethics, 121(3), 451-466. https://doi.org/10.1007/s10551-013-1732-0

Peña, M. L. y Pineda, L. E. (2013). Calidad de la gestión del sistema de información de una IPS de tercer nivel. Bogotá DC, Colombia. Cuadernos Latinoamericanos de Administración, 9(17), 58-71. Disponible en https://revistas.unbosque.edu.co/index.php/ cuaderlam/article/view/1239

Phillips, J. J. (2012). Return on investment in training and performance improvement programs. London: Routledge.

Pinilla, J., Martínez, J. y Cabarcas J. (2017). Capacidades de innovación del sector salud en Barranquilla: un análisis prospectivo a 2025. Economía y Región, 11(1), 127-154. Disponible en https://revistas.utb.edu.co/index.php/economiayregion/article/view/152

Ramazani, J. \& Jergeas, G. (2015). Project managers and the journey from good to great: The benefits of investment in project management training and education. International Journal of Project Management, 33(1), 41-52. https://doi.org/10.1016/j.ijproman.2014.03.012

República de Colombia. Congreso de la República. (23 de diciembre de 1993). Por el cual se crea el sistema de seguridad social integral y se dictan otras disposiciones. [Ley 100]. Diario Oficial No. 41.148. Recuperado de https://www.minsalud.gov.co/sites/rid/List.s/ BibliotecaDigital/RIDE/DE/DIJ/ley-100-de-1993.pdf 
República de Colombia. DNP. (2016). Salud Pública. [Online]. Disponible en: https://www. dnp.gov.co/programas/desarrollo-social/subdireccion-de-salud/Paginas/salud-publica. aspx

República de Colombia. MinSalud. (2016). Presupuesto 2016. Unidades Ejecutoras Ministerio de Salud: Proyectos de inversión. [Online]. Disponible en: https://www.minsalud. gov.co/sites/rid/Lists/BibliotecaDigital/RIDE/DE/PES/presupuesto-inversion-proyectos-2016.pdf

Rodelo, M. y Castro, S. (2016). Reflexiones sobre la educabilidad: elemento fundamental en el proceso educacional del desarrollo humano. Cultura Educación y Sociedad, 7(2), 94-104. Recuperado a partir de https://revistascientificas.cuc.edu.co/culturaeducacionysociedad/article/view/1104

Rodríguez-Villamizar, L., Vera-Cala, L., Rivera, R., Rojas, O. y Uribe, L. (2017). Evaluación de percepción de competencias de trabajadores respecto del Modelo Integral de Atención en Salud. Revista Salud UIS, 49(2), 302-311. http://dx.doi.org/10.18273/ revsal.v49n2-2017003

Ross, J. E. (2017). Total quality management: Text, cases, and readings. London: Routledge.

Serrano, L. y Ortíz, N. R. (2012). Caracterización del Nivel de desempeño en la Gestión por Procesos en IPS-Clínicas y Hospitales de Bucaramanga y Amb. Dyna, 79(175), 15-23. Disponible en https://revistas.unal.edu.co/index.php/dyna/article/view/25928

Storey, J. (2014). New Perspectives on Human Resource Management (Routledge Revivals). London: Routledge.

Topalović, S. (2015). The implementation of total quality management in order to improve production performance and enhancing the level of customer satisfaction. Procedia Technology, 19, 1016-1022. https://doi.org/10.1016/j.protcy.2015.02.145

Urdaneta, O. y Urdaneta, M. (2016). Evaluación del desempeño y motivación del personal en los Institutos de Investigaciones de Salud. Revista de Ciencias Sociales, 19(4), 672-682. Disponible en https://biblat.unam.mx/es/revista/revista-de-ciencias-sociales-universidad-del-zulia/articulo/evaluacion-del-desempeno-y-motivacion-del-personal-en-los-institutos-de-investigaciones-de-salud

Vom Brocke, J., Petry, M. \& Gonser, T. (2016). Business process management. In: Handbook of Business Transformation Management Methodology (pp. 137-172). London: Routledge.

Wandersman, A., Chien, V. H. \& Katz, J. (2012). Toward an evidence-based system for innovation support for implementing innovations with quality: tools, training, technical assistance, and quality assurance/quality improvement. American journal of community psychology, 50(3-4), 445-459. http://dx.doi.org/10.1007/s10464-012-9530-x

Remedios Pitre Redondo es Phd. en Ciencia con mención en Gerencia. Magister en Desarrollo y gestión de empresa sociales. Especialista en Pedagogía Economista. Docente Tiempo completo. Investigadora senior de Colciencias perteneciente al Grupo de investigación Tamaskal de la facultad de Ciencias sociales y Humanas. https://orcid.org/00000001-7373-1101 
Johennys Genith Brito Chiquillo es Magister en Cooperación Internacional y Gestión de Proyectos. https://orcid.org/0000-0002-1171-0177

Hugo Hernández Palma es Doctorante en Ingeniería Energética. Magister en Sistema de Gestión. Especialista en Estudios Pedagógicos. Especialista en Diseño y Evaluación de proyectos. Ingeniero Industrial. https://orcid.org/0000-0002-3873-0530 